\title{
Global sagittal alignment
}

\author{
Joseph H. Schwab ${ }^{1}$
}

Received: 21 July 2017 / Accepted: 1 August 2017 / Published online: 4 September 2017

(C) ISS 2017

Our understanding of sagittal alignment and its correlation with clinical outcomes such as pain and quality of life continues to evolve. What started out as research into the association of lumbar lordosis and radiographic parameters of the pelvis has broadened into global sagittal balance, which involves assessing the relative position of the cervical spine with the remainder of the skeleton down to the position of the ankles. The interest in these associations has been facilitated by full-length imaging modalities that allow one to accurately measure the relative position of these structures. There is a growing body of literature that attempts to correlate various measurements with clinical outcomes, and subsequently some of these measurements are now being used in surgical planning particularly in spinal surgery. However, advocates of global sagittal balance argue that alterations in any of the parameters that make up sagittal alignment will impact adjacent structures as these structures compensate for said changes. For that reason, while global sagittal alignment is most commonly discussed among clinicians who treat spinal pathology, the implications of sagittal alignment may impact and be impacted by pathology outside the spine. The purpose of this article is to introduce the concept of sagittal alignment along with some of the accepted radiographic parameters utilized in its measurement.

It is useful to begin a discussion on sagittal alignment with the pelvis since the pelvis dictates important aspects of alignment based on fixed anatomy. The first angle of interest is termed the pelvic incidence (PI), which is made by measuring

Joseph H. Schwab

jhschwab@mgh.harvard.edu

1 Department of Orthopaedic Surgery, Massachusetts General Hospital, 55 Fruit Street, Yawkey 3a, Boston, MA 02114, USA the angle between the perpendicular of the sacral endplate at the midpoint of the endplate with a line drawn from this point to the center of the femoral heads [1]. Since the sacral iliac joint does not allow significant motion, the PI is thought to be a fixed parameter. On the other hand, pelvic tilt (PT) is not a fixed angle, and it measures the relative rotation of the pelvis around the femoral heads. PT is made by drawing a vertical line from the center of the femoral head and measuring the angle between the vertical line and a line starting at the center of the femoral heads and ending at the midpoint of the sacral endplate. Rotating the pelvis around the femoral heads is one means by which the body compensates for alterations in sagittal alignment [1]. The sacral slope (SS) is the angle between a line drawn parallel to the sacral endplate and a horizontal line. The sacral slope (SS) is also mobile since it is position dependent [1]. PI, PT and SS are interrelated; however, PI is the only fixed parameter and therefore the most suitable for surgical planning. One can think of the PI as the cornerstone of sagittal alignment. Lumbar lordosis (LL) is not a fixed parameter; however, an individual's LL should correlate with their PI assuming they were born with normal sagittal alignment. This has proven to be useful in surgical planning for hypokyphosis of the lumbar spine. For instance, several studies have shown that, in normal subjects, the difference between the angle of the PI and LL should be less than 10. If one is planning on a surgery to correct the alignment of the lumbar spine then one should choose a method that will render the LL within $10^{\circ}$ of the PI. A different way of looking at this association is that if the difference between the PI and LL is greater than $10^{\circ}$ then there may be a sagittal mismatch. This is important because sagittal malalignment has been correlated with worse quality of life and more pain.

It is important to understand that one should not consider the lumbar lordosis and pelvic parameters in a vacuum. They are part of a chain that makes up global sagittal alignment. A 
patient's thoracic kyphosis that is measured by the Cobb angle between the 4th and 12th thoracic vertebrae is dependent upon the lumbar lordosis [2]. If the patient loses lumbar lordosis secondary to disc desiccation then they may try to compensate for that loss by developing secondary thoracic hypokyphosis and pelvic rotation to maintain sagittal alignment. While one should consider each part of the chain individually, it is also useful to use a more globally pertinent measurement such as the sagittal vertical axis (SVA) [1]. To measure the SVA one must measure the horizontal distance between two vertical lines. The first vertical line is drawn through the center of the seventh cervical vertebra, and the second vertical line is drawn through the posterior aspect of the sacral endplate. The SVA should be less than $4 \mathrm{~cm}$ in a healthy patient. While local pathology such as loss of LL may exist, the patient will often be able to compensate for this loss of lordosis. The compensation is designed to maintain the global sagittal alignment, which means maintaining the SVA. It is only after the compensatory mechanisms fail to maintain global sagittal alignment that the patient may become symptomatic. Our understanding of the compensatory mechanisms that the body uses to maintain alignment is evolving.

An example of the body's compensatory response to alterations in the chain of sagittal alignment includes knee flexion as measured by the knee angle (KA) [3]. The KA is measured by drawing a line down the mechanical axis of the tibia and another down the mechanical axis of the femur. An increase in KA reflects the body's response to a spinal pelvic mismatch. Another of the body's compensatory responses to sagittal malalignment is to shift the pelvis posteriorly. This is measured by the pelvic shift (P.Sh), which is the distance between two vertical lines. The first is a vertical line drawn through the posterior aspect of the sacral endplate, and the second is a vertical line drawn through the anterior aspect of the distal tibia [3]. This crouched position that we see in our patients and also in the public setting is a response to sagittal malalignment. Naturally, one can expect that shifting and rotating the pelvis will of course affect the position of the acetabulum [4]. This has implications for hip arthroplasty.

The angles and distances described above are age-dependent, and what is considered normal in a patient over 65 years would not be considered normal in a middle-aged person [5]. The concept of global sagittal alignment is evolving, and its importance seems to be growing. However, as with any evolving concept, one must apply the concepts with some restraint as the relative importance of these parameters becomes apparent.

\section{Compliance with ethical standards}

Conflict of interest I do not have any relevant conflict of interests.

\section{References}

1. Diebo BG, Varghese JJ, Lafage R, Schwab FJ, Lafage V. Sagittal alignment of the spine: What do you need to know? Clin Neurol Neurosurg. 2015;139:295-301.

2. Matsumoto H, Colacchio ND, Schwab FJ, Lafage V, Roye DP, Vitale MG. Flatback Revisited: Reciprocal Loss of Lumbar Lordosis Following Selective Thoracic Fusion in the Setting of Adolescent Idiopathic Scoliosis. Spine Deform. 2015;3(4):345-51

3. Ferrero E, Liabaud B, Challier V, Lafage R, Diebo BG, Vira S, et al. Role of pelvic translation and lowerextremity compensation to maintain gravity line position in spinal deformity. J Neurosurg Spine. 2016;24(3):436-46.

4. Buckland AJ, Vigdorchik J, Schwab FJ, Errico TJ, Lafage R, Ames C, et al. Acetabular Anteversion Changes Due to Spinal Deformity Correction: Bridging the Gap Between Hip and Spine Surgeons. J Bone Joint Surg Am. 2015;97(23):1913-20.

5. Jalai CM, Cruz DL, Diebo BG, Poorman G, Lafage R, Bess S, et al. Full-Body Analysis of Age-Adjusted Alignment in Adult Spinal Deformity Patients and Lower-Limb Compensation. Spine (Phila Pa 1976). 2017;42(9):653-61. 